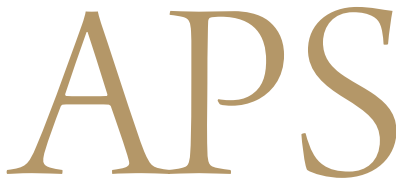

Archives of Plastic Surgery

\title{
Analysis of Scientific Papers Included in the Sciences Citation Index Expanded Written by South Korean Plastic Surgeons: 2001-2010
}

\author{
Ju Young Go ${ }^{1}$, Goo-Hyun Mun ${ }^{1}$, Byung-Joon Jeon ${ }^{1}$, So-Young Lim ${ }^{1}$, Jai-Kyong Pyon ${ }^{1}$, \\ Sa-Ik Bang ${ }^{1}$, Kap Sung Oh ${ }^{1}$, Myoung-Soo Shin ${ }^{2}$ \\ ${ }^{1}$ Department of Plastic Surgery, Samsung Medical Center, Sungkyunkwan University School of Medicine, Seoul; ${ }^{2}$ Department of Plastic \\ Surgery, Jeju National University Hospital, Jeju National University School of Medicine, Jeju, Korea
}

Background The purpose of our study was to analyze scientific papers published by South Korean plastic surgeons in journals included in the Science Citation Index Expanded (SCIE), and to evaluate the publication and research activities of Korean plastic surgeon.

Methods We conducted a survey of SCIE papers in the field of plastic surgery published by South Korean authors between 2001 and 2010 using Web of Science software. We further analyzed these results according to the number of publications per year, journals, institution, and type of papers. We also compared the total number of citations to published scientific papers. We analyzed the rank of South Korea among other countries in representative journals. Results Overall, 667 papers were published by South Korean authors between 2001 and 2010. The number of publications increased dramatically from $2003(n=31)$ to $2010(n=139)$. Subsequently, the ten most productive Korean medical colleges were identified. All published papers received 2,311 citations and the citation to paper ratio was 3.49. The rank of Korea among other countries in terms of the number of published papers remained in the top 10 during the recent 10 years.

Conclusions Publication output of Korean plastic surgeon over the last 10 years showed a remarkable growth in terms of quantity and quality. Currently, Korea is among the top six countries in representative plastic surgery journals. Korean plastic surgeons have played a central role in this progress, and it is anticipated that they will continue to do so in the future.

Keywords Republic of Korea / Surgery, plastic / Periodicals / Statistics
Correspondence: Myoung-Soo Shin Department of Plastic Surgery, Jeju National University Hospital, Jeju National University School of Medicine, 15 Aran 13-gil, Jeju 690-767, Korea

Tel: +82-64-717-1742

Fax: +82-64-757-8276

E-mail: ms57@unitel.co.kr
This article has been adapted from Ju Young Go's thesis submitted to Sungkyunkwan University Graduate School for the Master's degree in plastic surgery.

No potential conflict of interest relevant to this article was reported.

Received: 21 Jul 2011 • Revised: 1 Sep 2011 • Accepted: 6 Oct 2011

pISSN: 2234-6163 • elSSN: 2234-6171 • http://dx.doi.org/10.5999/aps.2012.39.1.46• Arch Plast Surg 2012;39:46-50

\section{INTRODUCTION}

The Korean Association of Plastic and Reconstructive Surgeons has played a central role in South Korean plastic surgery practices and research activities since its foundation in 1966. The num- ber of domestic and international research papers has steadily increased with an increasing number of members. In this study, we analyzed the scientific papers published by South Korean plastic surgeons listed in the Science Citation Index Expanded (SCIE) in order to evaluate the current level and status of their 
publication activities over the recent 10 years.

There are various methods for evaluating research achievements by individuals and groups. Among these, determining the number of published papers has been generally accepted as an objective method. In a quantitative evaluation of research results, the ability of the database to search for research papers is important. The citation index database is an essential tool to measure the degree of received citation $[1,2]$. The Science Citation Index (SCI) includes papers published worldwide in core journals in various fields of science and technology [3]. Therefore, domestic and foreign universities, research institutes, and research foundations prefer to examine the papers listed in Medline or the SCI for research performance evaluation. SCIE is an extension of the SCI available on CD-ROM. It is also provided as a web-based version that includes all SCI papers.

A 2001 study by Hwang et al. [4] confirmed the growth of the plastic surgery field in South Korea by analyzing the number of annual international publications on plastic surgery from 1974 to 2000 along with the differences between institutions and teaching hospitals. The present study aimed to identify the current quality and quantity of research performed by South Korean plastic surgeons. We also wanted to determine which medical institutions have the greatest number of publications in international journals over the past 10 years. Unlike previous publications which concentrated only on the analysis according to the first author, we reviewed each publication in detail by focusing on corresponding authors and analyzed research fields by plastic surgeons. Moreover, we aimed to evaluate the competence of South Korean plastic surgeons in research activities by comparing the number of research papers in representative international journals and ones published in South Korea.

\section{METHODS}

We conducted a survey of SCI papers in the field of plastic surgery by South Korean authors from 2001 to 2010. The ISI Web of Knowledge-Web of Science was used for our analysis; the search was conducted during the fourth week of March 2011. SCIE has the advantage of being updated on a weekly basis while the update of SCI is comparatively lagging due to the fact that it is provided on a CD-ROM. Accordingly, there was a case in which the SCI had a lower number of results than the SCIE for a publication with the same citation count in the journal [5]. Since the input of records for 2010 is still in progress at the time of our study, the data for 2010 does not fully reflect the total number of records.

Journals are classified into 172 research fields in the Web of Science (WoS) database [6]. Plastic surgery is included in the
Surgery category without being classified as an extra group in the SCIE. Research activities in the field of plastic surgery have become varied as seen in general and internal medicine as well as cell and tissue engineering. The selection of journals according to subject fields is limited by the fact that papers published in other journals are not searchable. Consequently, we performed our analysis by using the terms "South Korea", "plast", and "reconstr" in the address section on the preferential basis in order to minimize data omission. By doing this, we collected data by downloading information according to Authors, Title, Source, Address of Authors, and Times Cited. In this study, we limited our study to research publications by South Korean plastic surgeons along with reprint authors after identifying each record one by one. Research papers by South Korean authors were excluded from data if they belonged to foreign medical colleges or institutions.

SCIE has a technical problem in that many reprint authors are designated by the same initials and only one author is marked by a number of initials. We therefore personally classified results by reviewing original publications and investigating the authors' names in English. In cases where authors had moved to other organizations, their research papers were considered as ones which belong to the institution they were working when the paper was originally published. However, results were combined in the analysis of individual research achievement. The type of published research papers was cited according to the WoS classification. We excluded meeting abstracts and notes while articles, editorial materials, letters, proceedings papers, and reviews were included in our study.

To make minimize the number of omitted publications, we compared results by authors, organizations, papers, and year of publication. English names of South Korean medical colleges are taken from the Present Status of Korean Medical College Education. There are problems associated with the fact that the names of Korea medical institutions such as college of medicine was changed in the last 30 years and noted in different way in the journals. Thus, the tasks to bind many different names for the same institution as an institution were preceded. Finally, we selected the most representative five international plastic surgery journals and determined the rank of South Korea among other countries in terms of the number of published papers.

\section{RESULTS}

By using the SCIE database to confirm the whether the reprint authors were members of the Korean Association of Plastic and Reconstructive Surgeons, we found that a total of 667 papers were published between 2001 and 2010. Fifty-five papers were 
published in 2001. In 2003, however, only 31 papers were published. However, after this the number of publications consistently increased. The output in $2009(\mathrm{n}=96)$ was approximately three-times that in 2003, and the output in $2010(\mathrm{n}=139)$ was more than 4 times as great (Fig. 1).

Thirty-two medical college published papers listed in the SCIE and eight institutions published more than 30 SCIE papers. Additionally, 33 papers were published by authors from 23 plastic surgery clinics. As shown in Table 1, Inha University published the greatest number of papers (114) followed by Yonsei University (67), Korea University (59), Seoul National University (55), Catholic University (53), and the University of Ulsan (51).

One hundred and fifty-six authors published SCI papers as reprint authors. Among them, 12 authors published more than 10 SCI papers; the total number of papers published by these authors was 297 (44.53\%).

Most of the publications were articles (535 papers, $80.21 \%$ );

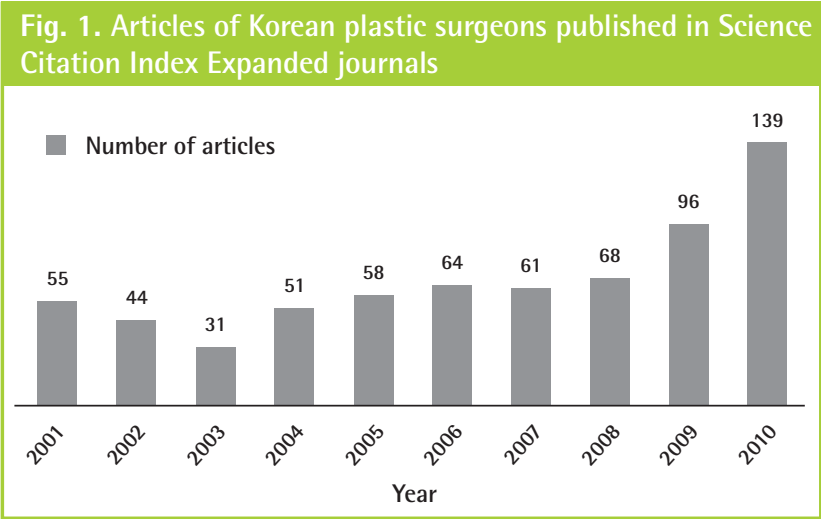

Table 1. Number of Science Citation Index Expanded papers listed by institution (2001-2010)

\begin{tabular}{|llcr|}
\hline Rank & \multicolumn{1}{c}{ Affiliation } & Frequency & Percent \\
\hline 1 & Inha University & 114 & 17.09 \\
2 & Yonsei University & 67 & 10.04 \\
3 & Korea University & 59 & 8.85 \\
4 & Seoul National University & 55 & 8.25 \\
5 & Catholic University & 53 & 7.95 \\
6 & University of Ulsan & 51 & 7.65 \\
7 & Kyungpook National University & 42 & 6.30 \\
8 & Sungkyunkwan University & 34 & 5.10 \\
9 & Chonnam National University & 23 & 3.45 \\
10 & Donga University & 16 & 2.40 \\
11 & Ewha Womans University & 14 & 2.10 \\
12 & Hallym University & 14 & 2.10 \\
13 & Hanyang University & 14 & 2.10 \\
14 & Keimyung University & 13 & 1.95 \\
- & 14 Institutions & 569 & 85.31 \\
- & 40 Others & 98 & 14.69 \\
& Total & 667 & 100.00 \\
\hline
\end{tabular}

three review papers were also published (Table 2). All published papers were cited 2,313 times and the citation to paper ratio was 3.47. Four hundred and thirty papers $(64.47 \%)$ were cited at least once (Table 3). Sixty-seven papers (10.0\%) were cited more than 10 times. The top five journals that published papers by South Korean authors (Table 4) were the Journal of Craniofacial Surgery $(\mathrm{n}=154)$, Plastic and Reconstructive Surgery $(\mathrm{n}=151)$, Annals of Plastic Surgery $(\mathrm{n}=118)$, Journal of Plastic Reconstructive and Aesthetic Surgery ( $\mathrm{n}=63)$, and Aesthetic Plastic Surgery $(\mathrm{n}=36)$. The number of papers published in these top five journals was 522 (78.3\%). The rank of South Korea among other countries in terms of published papers in these five journals has remained in the top 10 since 2001. When the period between 2001 and 2010 was taken into consideration, the publication percentage of South Korean authors was 3.2, and South Korea was the sixth in terms of the number of publications (Table 5, Fig. 2).

\section{DISCUSSION}

According to a 12-year survey conducted from 1988 to 1999 by Han and Lee [7] in 2000, 119 papers published in the field of plastic surgery field in Korean Medical College journals were listed in the Medline. Moreover, the total number of publications in journals indexed in the SCIE between 1974 and 2000

Table 2. Distribution of the types of documents published in journals listed in the Science Citation Index Expanded (20012010)

\begin{tabular}{|lc|}
\hline Type of document & No. (\%) \\
\hline Article & $535(80.21)$ \\
Editorial material & $59(8.85)$ \\
Letter & $48(7.20)$ \\
Proceedings paper & $22(3.30)$ \\
Review & $3(0.45)$ \\
\hline
\end{tabular}

Table 3. Distribution of cited Science Citation Index papers according to the number of times cited (2001-2010)

\begin{tabular}{|lcc|}
\hline No. of times cited & No. of papers & Citedness \\
\hline 1 & 93 & 93 \\
$2-5$ & 203 & 652 \\
$6-9$ & 67 & 480 \\
$10-15$ & 40 & 475 \\
$16-20$ & 14 & 245 \\
$21-30$ & 9 & 209 \\
$31-50$ & 4 & 159 \\
Subtotal & 430 & 2,313 \\
Not cited & 237 & - \\
Total & 667 & 2,313 \\
\hline
\end{tabular}


Table 4. Distribution of journals ranked by the number of articles published by South Korean plastic surgeons (2001-2010)

\begin{tabular}{|c|c|c|c|}
\hline Journal & Frequency & Percent & Cumulative percent \\
\hline Journal of craniofacial surgery & 154 & 23.09 & 23.09 \\
\hline Plastic and reconstructive surgery & 151 & 22.64 & 45.73 \\
\hline Annals of plastic surgery & 118 & 17.69 & 63.42 \\
\hline Journal of plastic reconstructive and aesthetic surgery & 63 & 9.45 & 72.86 \\
\hline Aesthetic plastic surgery & 36 & 5.40 & 78.26 \\
\hline Dermatologic surgery & 17 & 2.55 & 80.81 \\
\hline British journal of plastic surgery & 14 & 2.10 & 82.91 \\
\hline Tissue engineering and regenerative medicine & 13 & 1.95 & 84.86 \\
\hline Journal of Korean medical science & 11 & 1.65 & 86.51 \\
\hline Journal of reconstructive microsurgery & 10 & 1.50 & 88.01 \\
\hline Yonsei medical journal & 7 & 1.05 & 89.06 \\
\hline Burns & 5 & 0.75 & 89.81 \\
\hline Cleft palate-craniofacial journal & 4 & 0.60 & 90.40 \\
\hline Journal of oral and maxillofacial surgery & 4 & 0.60 & 91.00 \\
\hline $\begin{array}{l}\text { Scandinavian journal of plastic and reconstructive surgery and } \\
\text { hand surgery }\end{array}$ & 4 & 0.60 & 91.60 \\
\hline Journal of hand surgery-European volume & 3 & 0.45 & 92.05 \\
\hline Microsurgery & 3 & 0.45 & 92.50 \\
\hline Wound repair and regeneration & 3 & 0.45 & 92.95 \\
\hline Clinics in plastic surgery & 2 & 0.30 & 93.25 \\
\hline Journal of cranio-maxillofacial surgery & 2 & 0.30 & 93.55 \\
\hline Skin research and technology & 2 & 0.30 & 93.85 \\
\hline Surgical and radiologic anatomy & 2 & 0.30 & 94.15 \\
\hline 39 Others & 39 & 5.85 & 100.00 \\
\hline Total & 667 & 100.00 & - \\
\hline
\end{tabular}

Table 5 . Distribution by countries of papers published in five plastic surgery journals listed in the Science Citation Index Expanded (2001-2010)

\begin{tabular}{|lc|}
\hline Country & Percentage \\
\hline United States & 39.76 \\
Turkey & 9.08 \\
England & 7.98 \\
Japan & 5.35 \\
Italy & 5.21 \\
South Korea & 3.26 \\
Germany & 2.89 \\
Peoples R China & 2.69 \\
Brazil & 2.54 \\
Canada & 2.48 \\
Taiwan & 2.32 \\
\hline
\end{tabular}

was 195 based on the study by Hwang et al. [4]. In our study, we confirmed that the number of SCIE papers by plastic surgeons published in the last 10 years were three-times higher than in the previous 25 years. This finding was based on the results of quantitatively evaluating the research activities of South Korean plastic surgeons that measured the number of papers and times cited. More than 30 institutions and organizations had SCIE publications and there were eight institutions which published more than 30 papers. In-depth research achievements were pub-
Fig. 2. Charts showing changes in the rank of South Korea (2001-2010): out of 11 countries where papers published in five plastic journals listed in the Science Citation Index Expanded

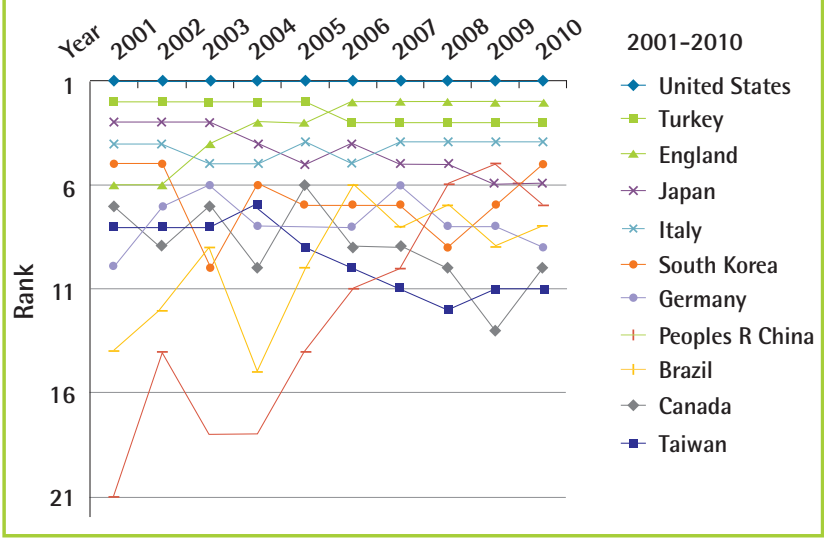

lished in more than 60 various SCIE journals research papers by corresponding authors in the field of cells and basic study of wounds including clinical techniques. We also found out that there were actively collaborative research projects among various institutions and departments. Moreover, there was marked quality increases which were estimated by the number of times the papers were cited as well as the increased number of publications.

A few technical problems were caused by searches for the 
number of times cited and ranks of countries using the WoS. One of these was that papers published a long time ago were generally cited more times while considerably recent publications were cited fewer times. Furthermore, the number of research papers from each country in WoS search included all nations listed for all the authors' addresses. Consequently, a number of papers were counted even though the authors were not South Korean reprint authors. Publications from authors in other fields such as otolaryngology were also included. Results from the searches could be different from data produced by limiting search items to reprint authors. This may cause the results to be less accurate. However, differences in the number of publications in five representative journals showed a similar pattern to results from searches performed according to reprint authors. The ranks of countries in the plastic surgery field can be determined based on this (Table 5).

The United States ranked first in the number of research papers in five representative journals with $40 \%$ of the total number of publications. This was followed by Turkey, the United Kingdom, and Italy with more than 5\% percent of the publications. South Korea was ranked after this. Compared to South Korea's faltered ranking in 2003 with a decreased number of research papers, we found that the ranking for South Korea has continuously increased since 2008 with a considerable surge in the number of publications. Most notably, although China was not among the top 20 in 2001, this country ranked higher than South Korea in between 2008 and 2009 by showing surprising growth rate (Fig. 2).

Through our statistical analysis, we determined that academic development of the plastic surgery field in South Korea has grown rapidly since 2000 . This development has increased at a fast rate, particularly over the last three years. Therefore, if the sustainable development is achieved in the future, it should be expected that South Korean surgeons will be a driving force in the field of plastic surgery.

\section{REFERENCES}

1. Meho LI, Sonnenwald DH. Citation ranking versus peer evaluation of senior faculty research performance: a case study of kurdish scholarship. J Am Soc Inf Sci 2000;51:12338.

2. Koenig ME. Bibliometric indicators versus expert opinion in assessing research performance. J Am Soc Inf Sci 1983; 34:136-45.

3. Thomson Reuters. Web of Science [Internet]. Philadelphia, PA: Thomson Reuters; 2011 [cited 2011 Mar 31]. Available from: http://apps.isiknowledge.com.

4. Hwang K, Kim CW, Lee CS, et al. Scientific publication productivity of Korean plastic surgeons: an analysis of 1974-2000 SCI papers. J Korean Soc Plast Reconstr Surg 2001;28:163-8.

5. Costas R, Iribarren-Maestro I. Variations in content and format of ISI databases in their different versions: the case of the Science Citation Index in CD-ROM and the Web of Science. Scientometrics 2007;72:167-83.

6. Thomson Reuters. Scope note 2011: Science Citation Index, Science Citation Index Expanded [Internet]. Philadelphia, PA: Thompson Reuters; 2011 [cited 2011 Mar 15]. Available from: http://scientific.thomsonreuters.com/mjl/scope/ scope_scie.

7. Han MC, Lee CS. Scientific publication productivity of Korean medical colleges: an analysis of 1988-1999 MEDLINE papers. J Korean Med Sci 2000;15:3-12. 